\title{
La coordinación de esfuerzos en casos clínicos complejos: un valor añadido de cuidados paliativos en un hospital universitario
}

\section{Coordinating efforts in complex clinical cases: added value of palliative care at a university teaching hospitalpcuth}

\author{
I. SanMiguel' ${ }^{1}$ A. Larumbe ${ }^{2}$, A. Chopitea ${ }^{3}$, C. Centeno ${ }^{2}$
}

\section{RESUMEN}

Los cuidados paliativos se entienden generalmente en el contexto de la atención a pacientes terminales ingresados en hospitales o en unidades de hospitales de larga estancia. Sin embargo, cada vez son más frecuentes estas unidades en hospitales de alto nivel de desarrollo.

Presentamos el caso de una paciente de especial complejidad, diagnosticada de carcinoma de recto en la que confluían cuatro problemas de difícil abordaje: dolor neuropático descontrolado a pesar de opioides, infección con repercusión sistémica, herida quirúrgica abierta y desánimo completo.

Mostramos las medidas que se adoptaron y cómo una excelente colaboración inter-departamental, bajo la coordinación del equipo de medicina paliativa, sirvió para solucionar una situación que en un punto llegó a parecer insostenible.

Palabras clave. Cuidados paliativos. Cuidados hospitalarios. Complejidad.

\begin{abstract}
Palliative care is generally understood on caring for terminal patients in chronic settings but more recently these units are developing also in acute care settings or university hospitals as consultants teams.

We report the case of a complex patient with rectal adenocarcinoma and four problems of difficult approach: uncontrolled neuropathic pain despite opioids treatment, systemic infection, depression with intense demoralisation and open surgery wound. We show the measures adopted and how an excellent inter-departmental collaboration under the co-ordination of palliative medicine consultant team helped to resolve the untenable situation.
\end{abstract}

Key words. Palliative care. Hospital care. Complexity.
1. Departamento de Oncología Radioterápica. Clinica Universidad de Navarra.

2. Unidad de Medicina Paliativa y Control de Síntomas. Clínica Universidad de Navarra.

3. Departamento de Oncología Médica. Clínica Universidad de Navarra.

Recepción: 27 de julio de 2010

Aceptación provisional: 4 de octubre de 2010

Aceptación definitiva: 29 de octubre de 2010

\section{Correspondencia}

Iñigo San Miguel

Departamento de Oncología Radioterápica

Clínica Universidad de Navarra

Avda. Pío XII, 36

31008 Pamplona. España.

E-mail: isanmiguel@unav.es 


\section{INTRODUCCIÓN}

Los cuidados paliativos se entienden generalmente en el contexto de la atención a pacientes terminales ingresados en hospitales o en unidades de hospitales de larga estancia. Sin embargo, cada vez son más frecuentes estas unidades en hospitales de alto nivel de desarrollo, bien para atender a pacientes con enfermedades avanzadas y síntomas complejos con alta carga emocional, con tratamientos oncológicos en curso o en estadios finales de la enfermedad, pero cuyo curso agudo no justifica el traslado a un hospital de larga estancia. En estas situaciones el equipo de cuidados paliativos debe hacer uso de una formación médica integral y mostrar la capacidad de establecer un marco de coordinación adecuado con otros especialistas ya que, a veces, hay que optar por medidas extraordinarias lejos del enfoque convencional de los cuidados paliativos.

Presentamos el caso de una paciente de especial complejidad, diagnosticada de carcinoma de recto en la que confluían cuatro problemas de difícil abordaje: dolor neuropático descontrolado a pesar de opioides, infección con repercusión sistémica, herida quirúrgica abierta y desánimo completo. En el presente trabajo se recogen las medidas adoptadas desde un enfoque inter-departamental.

\section{CASO}

Mujer de 53 años, con adenocarcinoma moderadamente diferenciado de recto uT3 $\mathrm{N}+$, tratada inicialmente con quimio-radioterapia con cirugía posterior (amputación abominoperineal) y braquiterapia perioperatoria hospitalizada.

La primera consulta al equipo de medicina paliativa se efectuó quince días después en el postoperatorio tardío por mal control del dolor. La paciente presentaba dolor en región anal, de intensidad moderada (EVN 4/10), con irradiación perineal que le impedía estar sentada. La valoración inicial detectó un componente de estrés emocional en relación con el control sintomático insuficiente y una pobre comunicación. Evaluación subjetiva de síntomas con el cuestionario $\mathrm{ESAS}^{1}$ : dolor $4 / 10$, cansancio $3 / 10$, náuseas $0 / 10$, depresión $5 / 10$, ansiedad $3 / 10$, somnolen- cia $3 / 10$, dificultad respiratoria $0 / 10$, anorexia $9 / 10$, dificultad para dormir $2 / 10$, malestar $6 / 10$. El dolor se controló en pocos días inicialmente con tramadol retard, $200 \mathrm{mg}$ cada $12 \mathrm{~h}$, lo que propició que continuara con quimioterapia ambulatoria. Sin embargo, en las semanas siguientes el dolor moderado volvió a ser problema por lo que, tras un mes de tratamiento con tramadol, se añadió gabapentina $300 \mathrm{mg}$ cada 8 horas y quince días después, en un ingreso, se sustituyó el tramadol por metadona que empezó titulándose con $5 \mathrm{mg}$ cada 8 horas y rescates de $2,5 \mathrm{mg}$. El dolor estaba controlado al cabo de pocos días con $15 \mathrm{mg}$ cada 8 horas.

Al cabo de diez semanas la paciente ingresó por exacerbación del dolor neuropático con intensidad máxima en zona sacra-perineal, depresión franca y un componente confusional. La situación de desánimo era absoluta tanto en la paciente como en su entorno familiar (ESAS): dolor $9 / 10$, cansancio $8 / 10$, náuseas $0 / 10$, depresión $7 / 10$, ansiedad $7 / 10$, somnolencia $8 / 10$, dificultad respiratoria $0 / 10$, anorexia $0 / 10$, dificultad para dormir $0 / 10$, malestar 9/10. En este momento su esperanza de vida era de más de tres meses, su estado funcional (Barthel) 85/100 y su estado general (Palliative Performance Status, PPS) 50/100. Se comenzó con perfusión intravenosa de ketamina con dosis de hasta $125 \mathrm{mg} /$ día y metadona $20 \mathrm{mg}$ cada $8 \mathrm{~h}$, sin lograr controlar el dolor insoportable. A las 24 horas del ingreso desarrolla cuadro febril con pérdida de la sutura perineal y una cavidad rectal contaminada con secreción verdosa, junto a leucocitosis. Un TAC evidenció una pequeña colección liquida presacra en la región intervenida.

En coordinación con los equipos de infecciosas y la Unidad de Dolor, se inicio antibioterapia de amplio espectro así como analgesia epidural (bupivacaína $0,5 \%$ + fentanilo en modo continuo con flujo de $6 \mathrm{ml} / \mathrm{h}$ y bolos de $2 \mathrm{mg}$ ), logrando un control del total del dolor (EVN 0/10) a las pocas horas. Ante la persistencia de fiebre y, dado que los cultivos de la herida habían resultado negativos, se programó drenaje guiado por TAC de la pequeña colección detectada. Se extrajeron $2 \mathrm{cc}$ de material aislándose cCitrobacter freundii. Se reorientó la antibioterapia lográndose el control del cuadro infeccioso al cabo de 4 días. Cuando la infección estuvo ya controlada, se sustituyó el catéter epidural por una bomba intratecal con morfina al $2 \%$ con un débito diario de $2 \mathrm{mg}$, manteniendo la analgesia total. Se inició tratamiento antidepresivo con mirtazapina y bromazepam.

Al cabo de tres meses de la intervención, como en la herida perineal no se apreciaban indicios de cicatrización a pesar de los cuidados del equipo quirúrgico, se solicitó valoración del 
equipo de cirugía plástica. Se aconsejó una intervención en un segundo tiempo con fines higiénicos, estéticos y para prevenir complicaciones. La cavidad rectal expuesta requería curas continuas y era motivo de malestar emocional de la paciente. Tras una estancia en su domicilio, la paciente fue reintervenida con nueva sutura. Regresó a casa sin dolor y con recuperación progresiva de su estado anímico y funcional, reiniciando de nuevo un régimen de quimioterapia ambulatoria un mes después de la reintervención.

Tras haber completado 3 ciclos de quimioterapia, desafortunadamente, en la reevaluación se comprobó progresión y la paciente decidió seguir tratamiento exclusivamente sintomático. En ese momento el estado general del paciente era claramente superior al de los momentos evolutivos previos.

El equipo de medicina paliativa del centro se encargó de contactar en su ciudad con profesionales de Medicina Paliativa que pudieran continuar su asistencia en la etapa final de su enfermedad. Fue también seguido telefónicamente por nuestro equipo. La paciente falleció en su domicilio con buen control sintomático.

\section{DISCUSIÓN}

La paciente aquí descrita estuvo inicialmente a cargo del Departamento de Oncología. Sin embargo, cuando el dolor incontrolado y el sufrimiento emocional pasaron a primer plano, se optó por la intervención del equipo de Medicina Paliativa que en nuestro centro cumple, entre otras, la función de equipo consultor de casos complejos, especialmente cuando convergen dolor, negatividad o se valoran intervenciones potencialmente extraordinarias en pacientes con limitada esperanza de vida, asumiendo en estas situaciones la responsabilidad de la coordinación. La paciente continuó a cargo de su oncólogo habitual. El equipo de consultores se suma a su asistencia con visita médica diaria, visita de enfermera dos veces al día y revisión diaria de la prescripción e intervención emocional y familiar diaria. De este modo la intervención de diversos equipos fue posible sin ninguna dificultad de coordinación.

Las características del dolor (localización, intensidad, componente neuropático, etc.) sugerían como causa la afectación del plexo presacro que tantas veces se aprecia en tumores rectales. Entre las etiologías potenciales de esa afectación podría estar la persistencia de enfermedad (de hecho se aplicó braquiterapia perioperatoria por la presencia de márgenes afectos), la recidiva tumoral (no visible en el TAC practicado) o una neuropatía rádica (había recibido radioterapia externa más braquiterapia perioperatoria). Debe reseñarse el empleo previo de fármacos como gabapentina, metadona y ketamina que, aunque han demostrado eficacia en situaciones de dolor neuropático, aquí fueron de escaso valor. Por el curso del dolor y por el ritmo de escalada de opioides requerido, no parece que hubiera un componente de hiperalgesia ${ }^{2}$. Bajo la hipótesis de que el dolor era debido fundamentalmente a un componente persistente de lesión estructural nerviosa, se optó por la vía espinal dado que el dolor pélvico era muy localizado, lo que a la luz de los resultados demostró ser una excelente opción.

Una pregunta relevante es si, en un caso como el presente, el equipo de cuidados paliativos aportó soluciones proporcionadas. Tanto el empleo de metadona ${ }^{3}$ como el uso de ketamina ${ }^{4}$ (en un caso de dolor neuropático) son medidas adecuadas en cuidados paliativos. El recurso a la vía espinal, bien sea con bomba epidural o con intratecal, aunque sólo se utilizan en escasas ocasiones para controlar el dolor ${ }^{5}$, son armas que deben tenerse en cuenta en situaciones como la presente. Por último, el drenaje guiado por TAC de la colección sospechosa $^{6}$, así como la realización de cirugía plástica con fines preventivos, higiénicos, estéticos o psicológicos, están plenamente justificadas si ello contribuye a aliviar los síntomas, mejorar la autoestima y en definitiva resolver los problemas importantes para el paciente. Es importante reseñar que, cuando se tomaron estas últimas medidas, no había signos de progresión de la enfermedad, hecho que desgraciadamente se objetivó tras el tercer ciclo de tratamiento. En ese momento, la última intervención de paliativos fue, de común acuerdo con la paciente, facilitar continuidad de cuidados en su ciudad de origen y seguir su evolución telefónicamente. 
El caso que nos ocupa refleja el papel de un equipo de paliativos en casos complejos en los que el estrés emocional, el dolor y la negación pueden dificultar o incluso imposibilitar la toma de otras decisiones terapéuticas necesarias, ayudando a la interlocución con la enferma y ayudando a jerarquizar problemas y a tomar decisiones.

\section{BIBLIOGRAFÍA}

1. Centeno C, Noguera A, López B, Carvajal A. Some tools of assessment used in Palliative Care (I): the edmonton system assessment symptoms (ESAS). Med Pal 2004; 11: 239-245.

2. Mercadante S, Ferrera P, Villari P, Arcuri E. Hyperalgesia: an emerging iatrogenic syndrome. J Pain Symptom Manage 2003; 26: 769775 .
3. Bruera E, Sweeney C. Methadone use in cancer patients with pain: a review. J Palliat Med 2002; 5: 127-138.

4. Fitzgibbon EJ, Viola R. Parenteral ketamine as an analgesic adjuvant for severe pain: development and retrospective audit of a protocol for a palliative care unit. J Palliat Med 2005; 8: 49-57.

5. Smith TJ, Coyne PJ, StaAts PS, Deer T, Stearns LJ, RAUCK RL et al. An implantable drug delivery system (IDDS) for refractory cancer pain provides sustained pain control, less drug-related toxicity, and possibly better survival compared with comprehensive medical management (CMM). Ann Oncol 2005; 16: 825-833.

6. Aubé C, Haghbin H, Lebigot J, Pessaux P, RidereauZins C, ARnAud JP et al. Value of the transgluteal approach under CT-guidance for percutaneous interventional image guided procedures. J Radiol 2004; 85 : 117-123. 